\title{
Two-Dimensional Magnetotelluric Modelling by the Boundary Element Method
}

\author{
Shi-zhe $\mathrm{XU}^{1}$ and Sheng-kai $\mathrm{ZHAO}^{2}$ \\ 'Shandong College of Oceanology, Qingdao, China \\ ${ }^{2}$ China University of Science and Technology, Hefei, China
}

(Received December 18, 1986; Revised July 15, 1987)

\begin{abstract}
2-D magnetotelluric modelling is usually done by the finite element method and the finite difference method. When the anomalous body is of a complicated shape, division of the region into cells and initial data preparation are rather tedious. In this paper, the boundary element method is applied to 2-D magnetotelluric models. First, we convert the boundary value problem of electromagnetic field into the boundary integral equation. Then, we solve the integral equation with the boundary element method. The boundaries of the domain considered and the anomalous body are divided into elements. The integral along all boundaries is divided into a sum of integrals along each element. Linear interpolation and Gaussian quardrature formula are used to calculate these integrals. After these steps, the integral equation is converted into a set of linear equations. Solving the equation system with the Gaussian elimination method, the electromagnetic field can be obtained and the apparent resistivity can be derived. The boundary element method is suitable for a model with one anomalous body. When there are several anomalous bodies, formula derivation and programing are complicated. This method is applied to two examples. The results agree well with those obtained by the finite element method.
\end{abstract}

\section{Introduction}

In the past three decades, two-dimensional magnetotelluric modelling has recieved high attention. In order to get solutions, various methods have been used. BRYNELLI et al. $(1963,1969)$ and DOSSO (1966) used an electrolytic tank. TAHERI (1968) used RC-network. D'ERCEVILlE and KUNETS (1962) and RANKIN (1962) found analytical solutions for some specific models. COGGON (1971), REDDY and RANKIN (1973), ZHAO and XU (1983) and XU and ZHAO (1985) used the finite element method. DMITRIYEV and ZAKHAROV (1970) presented a method for reducing the boundary value problem of electrodynamics to integral equations. PARRY and WARD (1971) used the integral equation method to solve a 2-D electromagnetic scattering problem. ZAKHAROV (1975) applied the integral equation method to 2-D magnetic and magnetotelluric modelling.

In this paper, the boundary element method is used to solve the integral equations for $H$ (or $E$ ) and its normal derivative. When the model consists of one anomalous body within a homogeneous space, this method is especially preferable. 
Compared with the finite element method, the boundary element method is simpler in element division and initial data preparation and requires less memory space. Two examples illustrate that the results of the boundary element method agree well with those of the finite element method. Although Green's theorem is used, there is something different from the method used by PARRY and WARD (1971) and ZAKHAROV (1975), such as the integral of a Delta function on a boundary point.

\section{Boundary Value Problem in Magnetotellurics}

Let the $x$-axis be aligned in the direction of strike, the $y$-axis orthogonal to the $x$-axis and the $z$-axis vertically downward. Assuming that time factor is $\mathrm{e}^{-i \omega t}$, the magnetotelluric apparent resistivity is expressed (CAGNIARD, 1953) for the $H_{x}$ polarization as

$$
\rho_{y x}=\frac{1}{\omega \mu}\left|\frac{E_{y}}{H_{x}}\right|^{2}, \quad \text { where } \quad E_{y}=\frac{1}{\sigma} \frac{\partial H_{x}}{\partial z}
$$

and for the $E_{x}$ polarization as

$$
\rho_{x y}=\frac{1}{\omega \mu}\left|\frac{E_{x}}{H_{y}}\right|^{2}, \quad \text { where } \quad H_{y}=\frac{1}{i \omega \mu} \frac{\partial E_{x}}{\partial z} .
$$

$\mu$ is the magnetic permeability and $\sigma$ the conductivity. In order to derive the apparent resistivities $\rho_{x y}$ and $\rho_{y x}$, we must calculate $H_{x}, E_{x}$ and their normal derivatives $\partial H_{x} / \partial z$ and $\partial E_{x} / \partial z$.

The governing equations for $H_{x}$ and $E_{x}$ are

$H_{x}$ polarization:

$$
\begin{aligned}
& \frac{\partial E_{z}}{\partial y}-\frac{\partial E_{y}}{\partial z}=i \omega \mu H_{x}, \\
& \frac{\partial H_{x}}{\partial z}=(\sigma-i \omega \varepsilon) E_{y} \\
& -\frac{\partial H_{x}}{\partial y}=(\sigma-i \omega \varepsilon) E_{z}
\end{aligned}
$$

$E_{x}$ polarization:

$$
\begin{aligned}
& \frac{\partial H_{z}}{\partial y}-\frac{\partial H_{y}}{\partial z}=(\sigma-i \omega \varepsilon) E_{x}, \\
& \frac{\partial E_{x}}{\partial z}=i \omega \mu H_{y}, \\
& -\frac{\partial E_{x}}{\partial y}=i \omega \mu H_{z},
\end{aligned}
$$


where $\varepsilon$ is the dielectric constant.

Substituting $E_{y}, E_{z}$ and $H_{y}, H_{z}$ from the second and third equations of (1.3) and (1.4) into the first equations of (1.3) and (1.4), we obtain the equations governing $H_{x}$ and $E_{x}$ in a homogeneous medium as

$$
\begin{aligned}
& \frac{\partial^{2} H_{x}}{\partial y^{2}}+\frac{\partial^{2} H_{x}}{\partial z^{2}}+\left(\omega^{2} \mu \varepsilon+i \omega \mu \sigma\right) H_{x}=0 \\
& \frac{\partial^{2} E_{x}}{\partial y^{2}}+\frac{\partial^{2} E_{x}}{\partial z^{2}}+\left(\omega^{2} \mu \varepsilon+i \omega \mu \sigma\right) E_{x}=0 .
\end{aligned}
$$

Rewriting (1.5) and (1.6) in a single form,

$$
\nabla^{2} U+k^{2} U=0
$$

where $U$ represents $H_{x}$ or $E_{x}, \nabla^{2}$ is the 2-dimensional Laplace operator and $k^{2}=$ $\omega^{2} \mu \varepsilon+i \omega \mu \sigma$. In free space, $\sigma=0, k^{2}=\omega^{2} \mu \varepsilon$. In conductive rock at frequencies used in magnetotellurics, we have $k^{2} \approx i \omega \mu \sigma$, since $\sigma / \omega \varepsilon \gg 1$.

Because the boundary conditions for $E_{x}$ and $H_{x}$ modes are different, we consider them separately.

\subsection{Boundary condition for $H_{x}$ polarization}

2.1.1. Interface boundary condition

Let $H_{x}^{(1)}, H_{x}^{(2)}$ represent the magnetic field $H_{x}$ on the two sides of an interface $\Gamma_{\mathrm{I}}$. From the continuity of tangential components, we have

$$
\left.H_{x}^{(1)}\right|_{\Gamma_{1}}=\left.H_{x}^{(2)}\right|_{\Gamma_{1}}
$$

From Fig. 1, the tangential component of the electric field on $\Gamma_{I}$ is

$$
\begin{aligned}
E_{\mathrm{t}} & =E_{y} \sin \alpha-E_{z} \cos \alpha \\
& =\frac{1}{\sigma}\left(\frac{\partial H_{x}}{\partial z} \sin \alpha+\frac{\partial H_{x}}{\partial y} \cos \alpha\right) \\
& =\frac{1}{\sigma} \frac{\partial H_{x}}{\partial n},
\end{aligned}
$$

where $\alpha$ is the angle between the normal vector $\boldsymbol{n}$ and the $y$-axis. From the continuity of the tangential component of the electric field we have

$$
\left.\frac{1}{\sigma_{1}} \frac{\partial H_{x}^{(1)}}{\partial n}\right|_{\Gamma_{1}}=\left.\frac{1}{\sigma_{2}} \frac{\partial H_{x}^{(2)}}{\partial n}\right|_{\Gamma_{1}} .
$$




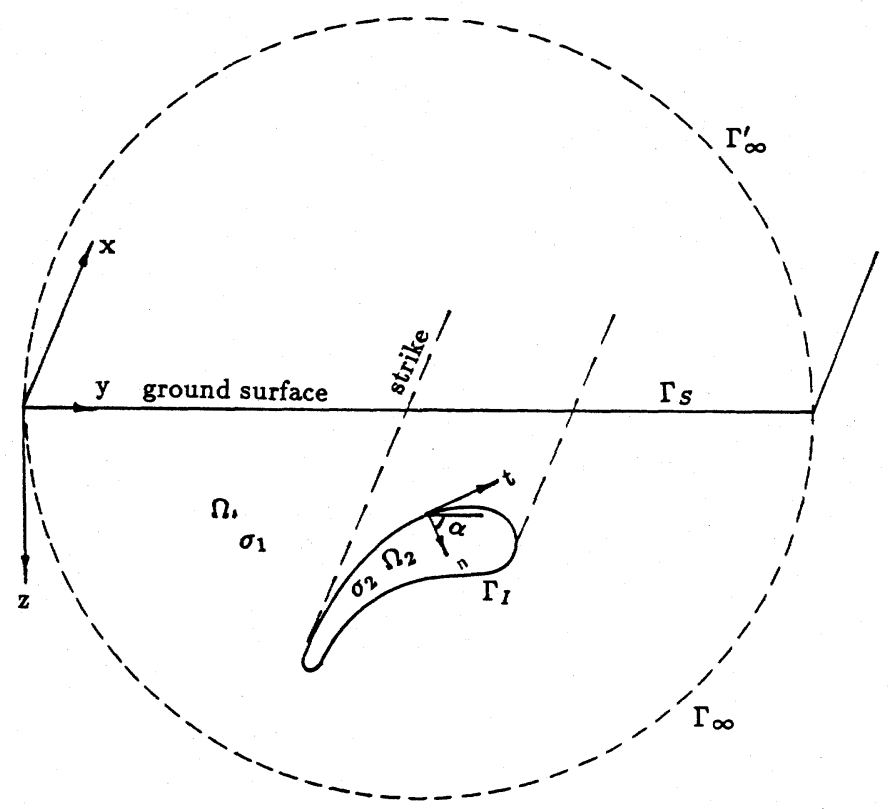

Fig. 1. The region and the boundary.

\subsubsection{Outside boundary condition}

Let $\sigma_{1}$ represent the conductivity of the medium around the anomalous body and $\sigma_{2}$ represent the conductivity of the anomalous body. We draw a semicircle $\Gamma_{\infty}$ with a very large radius under the ground surface $\Gamma_{\mathrm{S}}$ (Fig. 1). $\Gamma_{\infty}$ and $\Gamma_{\mathrm{S}}$ consist of a closed outside boundary surface $\Gamma\left(\Gamma=\Gamma_{\mathrm{S}}+\Gamma_{\infty}\right)$. From (1.3), $\partial H_{x} / \partial y=\sigma E_{y}$ and $\partial H_{x} / \partial y=-\sigma E_{z}$ in conductive rock. In free space, $\partial H_{x} / \partial z=-i \omega \varepsilon E_{y}$ and $\partial H_{x} / \partial y=i \omega \varepsilon E_{z}$. Because $\sigma / \omega \varepsilon \gg 1$, we may approximate $\partial H_{x} / \partial z=\partial H_{x} / \partial y=0$ at the free space side. This means that $H_{x}$ is constant regardless of the distribution of the subsurface conductivity. We set $H_{x}=1$ on $\Gamma_{\mathrm{s}}$. Because $\Gamma_{\infty}$ is far from the anomalous body, the electromagnetic field is the same as that for a homogeneous half space:

$$
\left.H_{x}\right|_{\Gamma_{\infty}}=\mathrm{e}^{i \sqrt{i \omega \mu \sigma_{1} z}}
$$

\subsection{The boundary condition for $E_{\mathrm{x}}$ polarization}

\subsubsection{Inside boundary condition}

Let $E_{x}{ }^{(1)}, E_{x}{ }^{(2)}$ represent the electric fields on the two sides of a boundary. From the continuity of the tangential components of electric fields, we have

$$
\left.H_{x}^{(1)}\right|_{\Gamma_{1}}=\left.E_{x}^{(2)}\right|_{\Gamma_{1}}
$$


From Fig. 1, the tangential component of $H$ is

$$
\begin{aligned}
H_{\mathrm{t}} & =H_{y} \sin \alpha-H_{z} \cos \alpha \\
& =\frac{1}{i \omega \mu}\left(\frac{\partial E_{x}}{\partial z} \sin \alpha+\frac{\partial E_{x}}{\partial y} \cos \alpha\right) \\
& =\frac{1}{i \omega \mu} \frac{\partial E_{x}}{\partial n} .
\end{aligned}
$$

We assume $\mu=\mu_{0}$, where $\mu_{0}$ is the permeability of free space. From the continuity of tangential magnetic field components, we have

$$
\left.\frac{\partial E_{x}^{(1)}}{\partial n}\right|_{\Gamma_{1}}=\left.\frac{\partial E_{x}^{(2)}}{\partial n}\right|_{\Gamma_{1}} .
$$

\subsubsection{Outside boundary condition}

Since $\Gamma_{\infty}$ is far from the anomalous body, the electric field on $\Gamma_{\infty}$ is the same as that in a homogeneous earth:

$$
\left.H_{x}\right|_{\Gamma_{\infty}}=\mathrm{e}^{i \sqrt{i \omega \mu \sigma_{1} Z}} .
$$

We draw a semi-circle $\Gamma_{\infty}^{\prime}$ with a very large radius (Fig. 1$) . \Gamma_{\infty}^{\prime}$ and $\Gamma_{\infty}$ constitute a closed outside surface. At $\Gamma_{\infty}^{\prime}$, the anomalous field due to the anomalous body is zero. $E_{x}$ consists of an incident field and a reflected field:

$$
\left.E_{x}\right|_{\Gamma_{\infty}^{\prime}}=E_{\mathrm{i}} \mathrm{e}^{i \sqrt{\omega^{2} \mu \varepsilon z}}+E_{\mathrm{r}} \mathrm{e}^{-i \sqrt{\omega^{2} \mu \varepsilon z}} .
$$

The first term is the incident wave and the second the reflected wave. $E_{\mathrm{i}}$ and $E_{\mathrm{r}}$ are coefficients to be determined. According to the boundary conditions at $z=0$ and $y= \pm \infty$, we find

$$
\begin{aligned}
& E_{\mathrm{i}}=\frac{1}{2}\left[1+(1+i) \sqrt{\frac{\sigma_{1}}{2 \omega \varepsilon}}\right], \\
& E_{\mathrm{r}}=\frac{1}{2}\left[1-(1+i) \sqrt{\frac{\sigma_{1}}{2 \omega \varepsilon}}\right] .
\end{aligned}
$$

Substituting $E_{\mathrm{r}}$ and $E_{\mathrm{i}}$ into (1.14), we derive

$$
\begin{aligned}
\left.E_{x}\right|_{\Gamma_{\infty}^{\prime}} & =\frac{1}{2}\left[1+(1+i) \sqrt{\frac{\sigma_{1}}{2 \omega \varepsilon}}\right] \mathrm{e}^{i \sqrt{\omega^{2} \mu \varepsilon z}}+\frac{1}{2}\left[1-(1+i) \sqrt{\frac{\sigma_{1}}{2 \omega \varepsilon}}\right] \mathrm{e}^{-i \sqrt{\omega^{2} \mu \varepsilon z}} \\
& =\cos (\omega \sqrt{\mu \varepsilon} z)-(1-i) \sqrt{\frac{\sigma_{1}}{2 \omega \varepsilon}} \sin (\omega \sqrt{\mu \varepsilon} z)
\end{aligned}
$$


Now we summarize the boundary value problem as follows. $H_{x}$ polarization:

Basic equation:

Inside boundary condition:

$$
\begin{aligned}
& \nabla^{2} U+k^{2} U=0, \\
& U^{(1)}=U^{(2)}, \\
& \frac{1}{\sigma_{1}} \frac{\partial U^{(1)}}{\partial n}=\frac{1}{\sigma_{2}} \frac{\partial U^{(2)}}{\partial n}, \\
& \left.U\right|_{\Gamma_{\mathrm{s}}}=1, \\
& \left.U\right|_{\Gamma_{\infty}}=\mathrm{e}^{i \sqrt{i \omega \mu \sigma_{1} z}} .
\end{aligned}
$$

Outside boundary condition: $\left.\quad U\right|_{\Gamma_{\mathrm{s}}}=1$,

$E_{x}$ polarization:

Basic equation:

$$
\begin{aligned}
& \nabla^{2} U+k^{2} U=0, \\
& U^{(1)}=U^{(2)}, \\
& \frac{\partial U^{(1)}}{\partial n}=\frac{\partial U^{(2)}}{\partial n},
\end{aligned}
$$

Inside boundary condition: $\quad U^{(1)}=U^{(2)}$,

Outside boundary condition:

$$
\left.U\right|_{\Gamma_{\infty}}=\mathrm{e}^{i \sqrt{i \omega \mu \sigma_{1} z}} .
$$

$$
\begin{aligned}
\left.U\right|_{\Gamma_{\infty}^{\prime}} & =\cos (\omega \sqrt{\mu \varepsilon} z), \\
& -(1-i) \sqrt{\frac{\sigma_{1}}{2 \omega \varepsilon}} \sin (\omega \sqrt{\mu \varepsilon} z) .
\end{aligned}
$$

Now our task is to find $U\left(H_{x}\right.$ or $\left.E_{x}\right)$ and its normal derivative $\partial U / \partial n$ on the ground surface.

\section{Green's Theorem and Fundamental Solution}

In the finite element method, the differential equations are transformed into a variation problem. Similarly, in the boundary element method, the differential equations are transformed into integral equations. This can be realized by using the Green's theorem:

$$
\int_{\Omega}\left(U \nabla^{2} \phi-\phi \nabla^{2} U\right) \mathrm{d} \Omega=\oint_{\Gamma}\left(U \frac{\partial \phi}{\partial n}-\phi \frac{\partial U}{\partial n}\right) \mathrm{d} \Gamma
$$

where $\Gamma$ is the boundary of domain $\Omega, \boldsymbol{n}$ is the outside normal vector, $U$ is a function to be solved and $\phi$ is the fundamental solution of the derivative equation:

$$
\nabla^{2} U+k^{2} U=0
$$

This means that $\phi$ is the Green's function of infinite space:

$$
\nabla^{2} \phi+k^{2} \phi=-\delta(P),
$$


where $P$ is an arbitrary point in region $\Omega$ (Fig. 2) and $\delta(P)$ is the Dirac delta function centered at $P$. Substituting (2.2), (2.3) into the left side of (2.1), we obtain

$$
\int_{\Omega}\left(U \nabla^{2} \phi-\phi \nabla^{2} U\right) \mathrm{d} \Omega=-\int_{\Omega} U \delta(P) \mathrm{d} \Omega .
$$

When $P$ is located on boundary $\Gamma$, from the property of the Dirac delta function (see Appendix),

$$
\int_{\Omega} U \delta(P) \mathrm{d} \Omega=\frac{\alpha_{\mathrm{p}}}{2 \pi} U(P)
$$

where $\alpha_{\mathrm{p}}$ is the angle subtended by $\Omega$ at $P$ (Fig. 2). Therefore,

$$
\frac{\alpha_{\mathrm{p}}}{2 \pi} U(P)=-\oint_{\Gamma}\left(U \frac{\partial \phi}{\partial n}-\phi \frac{\partial U}{\partial n}\right) \mathrm{d} \Gamma
$$

This is the integral equation that $U$ and its normal derivative $\partial U / \partial n$ at boundary points must satisfy. If $U$ on the boundary is given, we can find $\partial U / \partial n$ with the boundary element method. The fundamental solution of (2.2) is

$$
\phi=-\frac{1}{4} N_{0}(k r)
$$

where $N_{0}$ is the zero order Bessel function of the second kind, $r$ is the distance between point $P$ and an arbitrary point in the region, $k=\sqrt{\omega^{2} \mu \varepsilon}$ in free space and $k=\sqrt{i \omega \mu \sigma}$ below the ground surface.

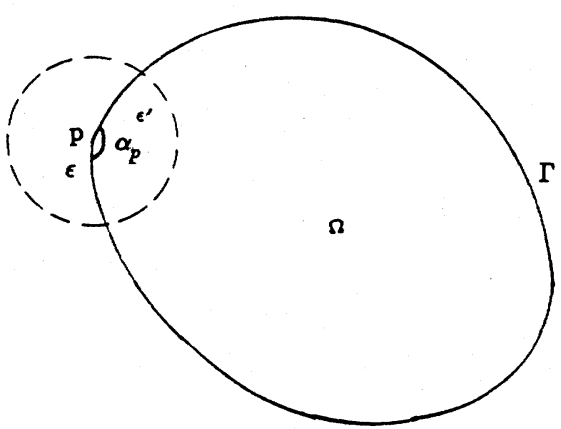

Fig. 2. Boundary point $P$ and angle $\alpha_{p}$ subtended by $\Omega$ at $P$. 


\section{Solution for $H_{x}$ Polarization}

\subsection{Integral equation}

Let $U^{(1)}$ and $U^{(2)}$ represent $H_{x}$ in $\Omega_{1}$ and $\Omega_{2}$ respectively (Fig. 3). For region $\Omega_{1}, U$ at boundary point $P$ is derived from (2.5) as

$$
\begin{aligned}
\frac{\alpha_{\mathrm{p}}}{2 \pi} U^{(1)}(P) & =-\oint_{\Gamma}\left(U^{(1)} \frac{\partial \phi}{\partial n}-\phi \frac{\partial U^{(1)}}{\partial n}\right) \mathrm{d} \Gamma \\
& =-\left(\int_{\Gamma_{\mathrm{s}}}+\int_{\Gamma_{\infty}}+\oint_{\Gamma_{1}}\right)\left(U^{(1)} \frac{\partial \phi}{\partial n}-\phi \frac{\partial U^{(1)}}{\partial n}\right) \mathrm{d} \Gamma
\end{aligned}
$$

The fundamental solution in $\Omega_{1}$ is $\phi=-(1 / 4) N_{0}\left(k_{1} r\right)$, where $k_{1}=\sqrt{i \omega \mu \sigma_{1}}$. Differentiating $\phi$ partially with respect to $n$, we get

$$
\frac{\partial \phi}{\partial n}=\frac{\partial \phi}{\partial r} \frac{\partial r}{\partial n}=\frac{1}{4} k_{1} N_{1}\left(k_{1} r\right) \cos \beta
$$

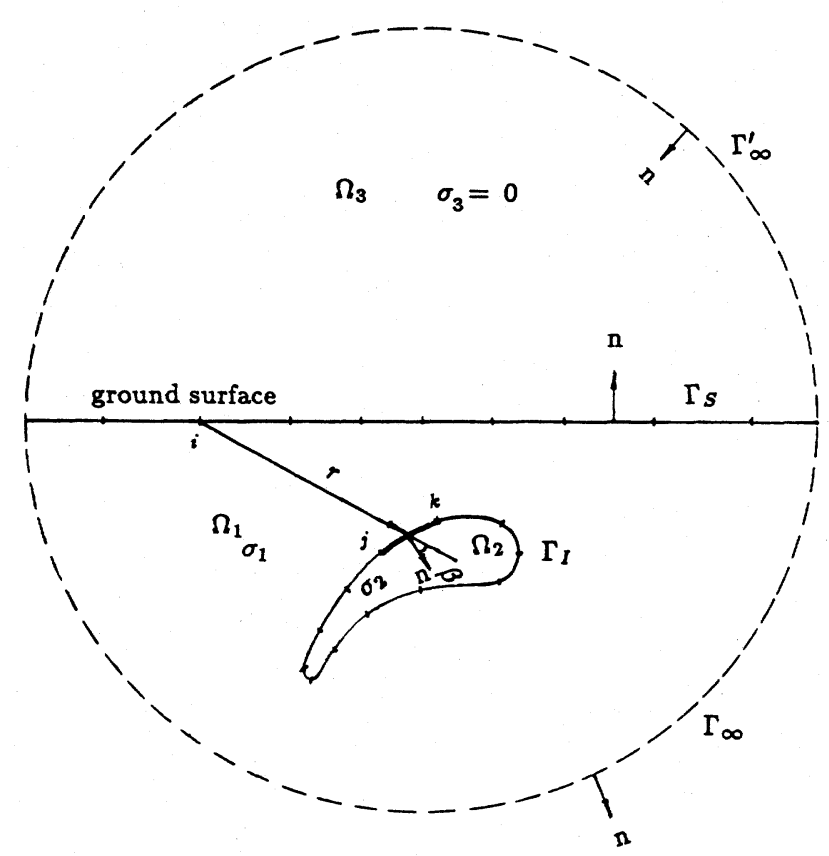

Fig. 3. Division of elements. 
where $N_{1}$ is the first order Bessel function of the second kind, $\beta$ is the angle between $r$ and $\boldsymbol{n}$ (Fig. 3).

At $\Gamma_{\infty}$, the anomalous electromagnetic field is zero. From (1.16),

$$
\begin{gathered}
\left.U^{(1)}\right|_{\Gamma_{\infty}}=\mathrm{e}^{i k_{1} z} \\
\left.\frac{\partial U^{(1)}}{\partial n}\right|_{\Gamma_{\infty}}=\left.\left(\frac{\partial U^{(1)}}{\partial z} \frac{\partial z}{\partial n}\right)\right|_{\Gamma_{\infty}}=i k_{1} \mathrm{e}^{i k_{1} z} \cos (n, z),
\end{gathered}
$$

where $(n, z)$ is the angle between $\boldsymbol{n}$ and $z$. So the integral on $\Gamma_{\infty}$ is

$$
\begin{aligned}
-\int_{\Gamma_{\infty}}\left(U^{(1)} \frac{\partial \phi}{\partial n}\right. & \left.-\frac{\partial U^{(1)}}{\partial n} \phi\right) \mathrm{d} \Gamma \\
& =-\int_{\Gamma_{\infty}} \frac{k_{1}}{4} \mathrm{e}^{i k_{1} z}\left[N_{1}\left(k_{1} r\right) \cos \beta+i N_{0}\left(k_{1} r\right) \cos (n, z)\right] \mathrm{d} \Gamma .
\end{aligned}
$$

We divide $\Gamma_{\infty}$ into elements, keeping the length of each element less than $\lambda_{1} / 8$, where $\lambda_{1}$ is the wave length in $\Omega_{1}$, and $\lambda_{1}=2 \sqrt{2} \pi / \sqrt{\omega \mu \sigma_{1}}$. Then we use Gaussian quadrature formula to integrate over each element (see (3.5)). We add together and denote as $C_{\mathrm{p}}$, which is a function of $P$. Equation (3.1) can be rewritten as

$$
\begin{aligned}
\frac{\alpha_{\mathrm{p}}}{2 \pi} U^{(1)}(P)= & -\int_{\Gamma_{\mathrm{s}}} U^{(1)} \frac{\partial \phi}{\partial r} \cos \beta \mathrm{d} \Gamma+\int_{\Gamma_{\mathrm{s}}} \frac{\partial U^{(1)}}{\partial n} \phi \mathrm{d} \Gamma \\
& -\oint_{\Gamma_{\mathrm{l}}} U^{(1)} \frac{\partial \phi}{\partial r} \cos \beta \mathrm{d} \Gamma+\int_{\Gamma_{1}} \frac{\partial U^{(1)}}{\partial n} \phi \mathrm{d} \Gamma+C_{\mathrm{p}}
\end{aligned}
$$

For region $\Omega_{2}, U^{(2)}(P)$ at point $P$ on $\Gamma_{\mathrm{I}}$ is derived from (2.5) as

$$
\left(1-\frac{\alpha_{\mathrm{p}}}{2 \pi}\right) U^{(2)}(P)=\oint_{\Gamma_{1}} U^{(2)} \frac{\partial \phi}{\partial r} \cos \beta \mathrm{d} \Gamma-\oint_{\Gamma_{1}} \frac{\partial U^{(2)}}{\partial n} \phi \mathrm{d} \Gamma_{3}
$$

where $\left(1-\alpha_{\mathrm{p}} / 2 \pi\right)$ is the angle subtended by $\Omega_{2}$ at $P$. Because the normal vector of $\Gamma_{\mathrm{I}}$ is defined from inward, the sign in front of the above integrals has been changed. The fundamental solution in region $\Omega_{2}$ is $\phi=-(1 / 4) N_{0}\left(k_{2} r\right)$, where $k_{2}=\sqrt{i \omega \mu \sigma_{2}}$. Substituting the boundary condition (1.16) into (3.3), we obtain

$$
\left(1-\frac{\alpha_{\mathrm{p}}}{2 \pi}\right) U^{(1)}(P)=\oint_{\Gamma_{1}} U^{(1)} \frac{\partial \phi}{\partial r} \cos \beta \mathrm{d} \Gamma-\oint_{\Gamma_{1}} \frac{\sigma_{2}}{\sigma_{1}} \frac{\partial U^{(1)}}{\partial n} \phi \mathrm{d} \Gamma .
$$

(3.2) and (3.4) are integral equations. Solving the equations with the boundary element method, we can find $\partial U / \partial n$ on ground surface $\Gamma_{\mathrm{S}}$. 


\subsection{Boundary element method}

We divide $\Gamma_{\mathrm{S}}$ and $\Gamma_{\mathrm{I}}$ into elements, approximate each element as a straight line segment (see Fig. 3) and make the length of element less than $\lambda_{1} / 8$ and $\lambda_{2} / 8$, where $\lambda_{1}$ and $\lambda_{2}$ are the wavelengths in $\Omega_{1}$ and $\Omega_{2}$, respectively. The ends of the element are nodes. There are $n$ nodes on $\Gamma_{\mathrm{s}}$, whose ordinal numbers are from 1 to $n$. There are $m-n$ nodes on $\Gamma_{\mathrm{I}}$, whose ordinal numbers are from $n+1$ to $m$. We divide the integral equation (3.2) into a sum of integrals on each element. For node $i$, equation (3.2) can be written as

$$
\begin{aligned}
\frac{\alpha_{i}}{2 \pi} U_{i}^{(1)}= & -\sum_{\Gamma_{\mathrm{s}}} \int_{\Gamma_{\mathrm{E}}} U^{(1)} \frac{k_{1}}{4} N_{\mathrm{l}}\left(k_{1} r\right) \cos \beta \mathrm{d} \Gamma-\sum_{\Gamma_{\mathrm{s}}} \int_{\Gamma_{\mathrm{E}}} \frac{\partial U^{(1)}}{\partial n} \frac{N_{0}\left(k_{1} r\right)}{4} \mathrm{~d} \Gamma \\
& -\sum_{\Gamma_{\mathrm{I}}} \int_{\Gamma_{\mathrm{E}}} U^{(1)} \frac{k_{1}}{4} N_{\mathrm{l}}\left(k_{1} r\right) \cos \beta \mathrm{d} \Gamma-\sum_{\Gamma_{1}} \int_{\Gamma_{\mathrm{E}}} \frac{\partial U^{(1)}}{\partial n} \frac{N_{0}\left(k_{1} r\right)}{4} \mathrm{~d} \Gamma+C_{i .} .
\end{aligned}
$$

The node numbers of the two ends of an element are taken as $j, k$ with coordinates $\left(x_{j}, y_{j}\right),\left(x_{k}, y_{k}\right)$. We assume that $U^{(1)}$ and $\partial U^{(1)} / \partial n$ are linear functions in each element. Using $U_{j}^{(1)}, U_{k}^{(1)},\left(\partial U^{(1)} / \partial n\right)_{j}$ and $\left(\partial U^{(1)} / \partial n\right)_{k}$ to represent $U^{(1)}$ and $\partial U^{(1)} / \partial n$ at $j$ and $k$, $U^{(1)}$ and $\partial U^{(1)} / \partial n$ can be written as

$$
\begin{gathered}
U^{(1)}=\xi_{j} U_{j}^{(1)}+\xi_{k} U_{k}^{(1)}=\left[\begin{array}{ll}
\xi_{j} & \xi_{k}
\end{array}\right]\left[\begin{array}{ll}
U_{j}^{(1)} & U_{k}^{(1)}
\end{array}\right]^{T}, \\
\frac{\partial U^{(1)}}{\partial n}=\xi_{j}\left(\frac{\partial U^{(1)}}{\partial n}\right)_{j}+\xi_{k}\left(\frac{\partial U^{(1)}}{\partial n}\right)_{k}=\left[\begin{array}{ll}
\xi_{j} & \xi_{k}
\end{array}\right]\left[\left(\frac{\partial U^{(1)}}{\partial n}\right)_{j}\left(\frac{\partial U^{(1)}}{\partial n}\right)_{k}\right]^{T},
\end{gathered}
$$

where $\xi_{j}, \xi_{k}$ are linear functions and defined as

$$
x=\xi_{j} x_{j}+\xi_{k} x_{k}, \quad y=\xi_{j} y_{j}+\xi_{k} y_{k} .
$$

It is obvious that at point $j, \xi_{j}=1, \xi_{k}=0$ and at point $k, \xi_{j}=0, \xi_{k}=1$.

The integral over an element can be derived as follows:

$$
\begin{aligned}
\int_{\Gamma_{\mathrm{E}}} U^{(1)} \frac{-k_{1} N_{1}\left(k_{1} r\right)}{4} \cos \beta \mathrm{d} \Gamma & =\int_{\Gamma_{\mathrm{E}}}\left[\begin{array}{ll}
\xi_{j} & \xi_{k}
\end{array}\right] \frac{-k_{1} N_{1}\left(k_{1} r\right)}{4} \cos \beta \mathrm{d} \Gamma \cdot\left[\begin{array}{ll}
U_{j}^{(1)} & U_{k}^{(1)}
\end{array}\right]^{T} \\
& =\left[\begin{array}{ll}
f_{i j} & f_{i k}
\end{array}\right]\left[\begin{array}{ll}
U_{j}^{(1)} & U_{k}^{(1)}
\end{array}\right]^{T}
\end{aligned}
$$

where

$$
\begin{gathered}
f_{i j}=\int_{\Gamma_{\mathrm{E}}} \xi_{j} \frac{-k_{1} N_{1}\left(k_{1} r\right)}{4} \cos \beta \mathrm{d} \Gamma, \\
f_{i k}=\int_{\Gamma_{\mathrm{E}}} \xi_{k} \frac{-k_{1} N_{1}\left(k_{1} r\right)}{4} \cos \beta \mathrm{d} \Gamma .
\end{gathered}
$$

All these integrals are complex and are accomplished with the Gaussian quadrature formula (BREBBIA, 1978). The integral over an element is derived similarly as 


$$
\begin{aligned}
\int_{\Gamma_{\mathrm{E}}} \frac{\partial U^{(1)}}{\partial n} \frac{-N_{0}\left(k_{1} r\right)}{4} \mathrm{~d} \Gamma & =\int_{\Gamma_{\mathrm{E}}}\left[\begin{array}{ll}
\xi_{j} & \xi_{k}
\end{array}\right] \frac{-N_{0}\left(k_{1} r\right)}{4} \mathrm{~d} \Gamma \cdot\left[\left(\frac{\partial U^{(1)}}{\partial n}\right)_{j}\left(\frac{\partial U^{(1)}}{\partial n}\right)_{k}\right]^{T} \\
& =\left[\begin{array}{ll}
d_{i j} & d_{i k}
\end{array}\right]\left[\left(\frac{\partial U^{(1)}}{\partial n}\right)_{j}\left(\frac{\partial U^{(1)}}{\partial n}\right)_{k}\right]^{T}
\end{aligned}
$$

where

$$
\begin{aligned}
& d_{i j}=\int_{\Gamma_{\mathrm{E}}} \xi_{j} \frac{-N_{0}\left(k_{1} r\right)}{4} \mathrm{~d} \Gamma, \\
& d_{i k}=\int_{\Gamma_{\mathrm{E}}} \xi_{k} \frac{-N_{0}\left(k_{1} r\right)}{4} \mathrm{~d} \Gamma .
\end{aligned}
$$

The elements containing node $i$ are singular, since $r \rightarrow 0, N_{0}\left(k_{\mathrm{i}} r\right) \rightarrow \infty$, and $N_{1}\left(k_{1} r\right) \rightarrow \infty$ in these elements. However, the integrals $f_{i j}, f_{i k}, d_{i j}$ and $d_{i k}$ on these elements are definite. For instance (see Fig. 4),

$$
\begin{aligned}
f_{i j} & =\int_{\Gamma_{\mathrm{E}}}-\xi_{j} \frac{k_{1}}{4} N_{1}\left(k_{1} r\right) \cos \beta \mathrm{d} \Gamma=0, \quad(\because \cos \beta=0) \\
d_{i j} & =\frac{1}{4} \int_{\Gamma_{\mathrm{E}}}-\xi_{j} N_{0}\left(k_{1} r\right) \mathrm{d} \Gamma \\
& \approx \int_{0}^{l} \frac{l-r}{l} \frac{1}{2 \pi} \ln \frac{k_{1} r}{2} \mathrm{~d} r, \quad\left(\because \xi_{j}=\frac{l-r}{l}, \lim _{r \rightarrow 0} N_{0}\left(k_{1} r\right)=-\frac{2}{\pi} \ln \frac{k_{1} r}{2}\right) \\
& =-\frac{l}{4 \pi}\left(\frac{3}{2}-\ln \frac{k_{1} r}{2}\right),
\end{aligned}
$$

where $l$ is the length of the element.

The sum of all integrals over each element on $\Gamma_{\mathrm{s}}$ is obtained as

$$
\begin{aligned}
& \sum_{\Gamma_{\mathrm{S}}} \int_{\Gamma_{\mathrm{E}}} U^{(1)} \frac{-k_{1} N_{1}\left(k_{1} r\right)}{4} \cos \beta \mathrm{d} \Gamma \\
& =\left[F_{i 1} \cdots F_{i j} \cdots F_{i n}\right]\left[U_{1}^{(1)} \cdots U_{j}^{(1)} \cdots U_{n}^{(1)}\right]^{T}=\boldsymbol{F}_{i \mathrm{~S}} \boldsymbol{U}_{\mathrm{S}}^{(1)}
\end{aligned}
$$

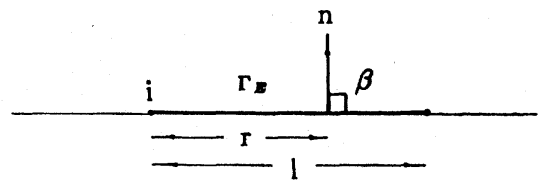

Fig. 4. Integral over the element containing node $i$. 


$$
\begin{aligned}
& \sum_{\Gamma_{\mathrm{S}}} \int_{\Gamma_{\mathrm{E}}} \frac{\partial U^{(1)}}{\partial n} \frac{-N_{0}\left(k_{1} r\right)}{4} \mathrm{~d} \Gamma \\
& =\left[D_{i 1} \cdots D_{i j} \cdots D_{i n}\right]\left[\left(\frac{\partial U^{(1)}}{\partial n}\right)_{1} \cdots\left(\frac{\partial U^{(1)}}{\partial n}\right)_{j} \cdots\left(\frac{\partial U^{(1)}}{\partial n}\right)_{n}\right]^{T}=\boldsymbol{D}_{i \mathrm{~S}}\left(\frac{\partial \boldsymbol{U}^{(1)}}{\partial \boldsymbol{n}}\right)_{\mathrm{S}},
\end{aligned}
$$

where

$$
\begin{gathered}
\boldsymbol{U}_{\mathrm{S}}^{(1)}=\left[U_{1}^{(1)} \cdots U_{n}^{(1)}\right]^{T} \\
\left(\frac{\partial \boldsymbol{U}^{(1)}}{\partial \boldsymbol{n}}\right)_{\mathrm{S}}=\left[\left(\frac{\partial U^{(1)}}{\partial n}\right)_{1} \cdots\left(\frac{\partial U^{(1)}}{\partial n}\right)_{n}\right]^{T}
\end{gathered}
$$

are the column vectors formed by $U^{(1)}$ and $\partial U^{(1)} / \partial n$ at each node on $\Gamma_{\mathrm{S}}, \mathrm{F}_{i j}$ and $D_{i j}$ are the sum of $f_{i j}, d_{i j}$ at the elements around node $j$ :

$$
\begin{aligned}
\boldsymbol{F}_{i \mathrm{~S}} & =\left[F_{i 1} \cdots F_{i j} \cdots F_{i n}\right], \\
\boldsymbol{D}_{i \mathrm{~S}} & =\left[D_{i 1} \cdots D_{i j} \cdots D_{i n}\right] .
\end{aligned}
$$

Only those elements on $\Gamma_{\mathrm{S}}$ have contribution to $\boldsymbol{F}_{i \mathrm{~S}}, \boldsymbol{D}_{i \mathrm{~s}}$.

Using the same method, we can obtain the sum of integrals over elements on $\Gamma_{I}$ as

$$
\begin{aligned}
& \sum_{\Gamma_{1}} \int_{\Gamma_{\mathrm{E}}} U^{(1)} \frac{-k_{1} N_{1}\left(k_{1} r\right)}{4} \cos \beta \mathrm{d} \Gamma=\left[F_{i(n+1)} \cdots F_{i m}\right]\left[U_{n+1}^{(1)} \cdots U_{m}^{(1)}\right]^{T}=\boldsymbol{F}_{i 1} \boldsymbol{U}_{\mathrm{I}}^{(1)} \\
& \sum_{\Gamma_{1}} \int_{\Gamma_{\mathrm{E}}} \frac{\partial U^{(1)}}{\partial n} \frac{-N_{0}\left(k_{1} r\right)}{4} \mathrm{~d} \Gamma \\
& =\left[D_{i(n+1)} \cdots D_{i m}\right]\left[\left(\frac{\partial U^{(1)}}{\partial n}\right)_{n+1} \ldots\left(\frac{\partial U^{(1)}}{\partial n}\right)_{m}\right]^{T}=\boldsymbol{D}_{i \mathrm{I}}\left(\frac{\partial \boldsymbol{U}^{(1)}}{\partial \boldsymbol{n}}\right)_{\mathrm{I}},
\end{aligned}
$$

where

$$
\begin{gathered}
\boldsymbol{U}_{\mathrm{I}}^{(1)}=\left[U_{n+1}^{(1)} \cdots U_{\mathrm{m}}^{(1)}\right]^{T} \\
\left(\frac{\partial \boldsymbol{U}^{(1)}}{\partial \boldsymbol{n}}\right)_{\mathrm{I}}=\left[\left(\frac{\partial U^{(1)}}{\partial n}\right)_{n+1} \ldots\left(\frac{\partial U^{(1)}}{\partial n}\right)_{m}\right]^{T}
\end{gathered}
$$

are column vectors formed by $U^{(1)}$ and $\partial U^{(1)} / \partial n$ of each node on $\Gamma_{\mathrm{I}}$ :

$$
\begin{aligned}
\boldsymbol{F}_{i \mathrm{I}} & =\left[F_{i(n+1)} \cdots F_{i m}\right], \\
\boldsymbol{D}_{i \mathrm{I}} & =\left[D_{i(n+1)} \cdots D_{i m}\right] .
\end{aligned}
$$


Only those elements on $\Gamma_{\mathrm{S}}$ have contribution to $\boldsymbol{F}_{i \mathrm{I}}, \boldsymbol{D}_{i \mathrm{I}}$.

Substituting (3.8)-(3.11) into (3.5), we obtain

$\frac{\alpha_{i}}{2 \pi} U_{\mathrm{i}}^{(1)}=\boldsymbol{F}_{i \mathrm{~S}} \boldsymbol{U}_{\mathrm{S}}^{(1)}+\boldsymbol{D}_{i \mathrm{~S}}\left(\frac{\partial \boldsymbol{U}^{(1)}}{\partial \boldsymbol{n}}\right)_{\mathrm{S}}+\boldsymbol{F}_{i \mathrm{I}} \boldsymbol{U}_{\mathrm{I}}^{(1)}+\boldsymbol{D}_{i \mathrm{I}}\left(\frac{\partial \boldsymbol{U}^{(1)}}{\partial \boldsymbol{n}}\right)_{\mathrm{I}}+C_{i}$.

For each node we can derive an equation as above. From all the nodes $i(1 \leqslant i \leqslant n)$ on $\Gamma_{\mathrm{s}}$, we derive an equation system:

$$
\frac{\alpha_{\mathrm{S}}}{2 \pi} \boldsymbol{U}_{\mathrm{S}}^{(1)}=\boldsymbol{F}_{\mathrm{SS}} \boldsymbol{U}_{\mathrm{S}}^{(1)}+\boldsymbol{D}_{\mathrm{SS}}\left(\frac{\partial \boldsymbol{U}^{(1)}}{\partial \boldsymbol{n}}\right)_{\mathrm{S}}+\boldsymbol{F}_{\mathrm{SI}} \boldsymbol{U}_{\mathrm{I}}^{(1)}+\boldsymbol{D}_{\mathrm{SI}}\left(\frac{\partial \boldsymbol{U}^{(1)}}{\partial \boldsymbol{n}}\right)_{\mathrm{I}}+C_{\mathrm{S}}
$$

From all the nodes $i(n+1 \leq i \leq m)$ on $\Gamma_{\mathrm{I}}$ we derive another equation system:

$$
\frac{\alpha_{\mathrm{I}}}{2 \pi} U_{\mathrm{I}}^{(1)}=\boldsymbol{F}_{\mathrm{IS}} \boldsymbol{U}_{\mathrm{S}}^{(1)}+\boldsymbol{D}_{\mathrm{IS}}\left(\frac{\partial \boldsymbol{U}^{(1)}}{\partial \boldsymbol{n}}\right)_{\mathrm{S}}+\boldsymbol{F}_{\mathrm{II}} \boldsymbol{U}_{\mathrm{I}}^{(1)}+\boldsymbol{D}_{\mathrm{II}}\left(\frac{\partial \boldsymbol{U}^{(1)}}{\partial \boldsymbol{n}}\right)_{\mathrm{I}}+C_{\mathrm{I}},
$$

where

$$
\begin{gathered}
\alpha_{\mathrm{S}}=\operatorname{diag}\left[\alpha_{1} \cdots \alpha_{i} \cdots \alpha_{n}\right] \\
\boldsymbol{F}_{\mathrm{SS}}=\left[F_{1 \mathrm{~S}} \cdots F_{i \mathrm{~s}} \cdots F_{n \mathrm{~s}}\right]^{T} \\
\boldsymbol{D}_{\mathrm{SS}}=\left[D_{1 s} \cdots D_{i \mathrm{~S}} \cdots D_{n \mathrm{~S}}\right]^{T} \\
\boldsymbol{F}_{\mathrm{SI}}=\left[F_{11} \cdots F_{i \mathrm{I}} \cdots F_{n \mathrm{I}}\right]^{T} \\
\boldsymbol{D}_{\mathrm{SI}}=\left[D_{11} \cdots D_{i 1} \cdots D_{n \mathrm{I}}\right]^{T} \\
\boldsymbol{C}_{\mathrm{S}}=\left(C_{1} \cdots C_{i} \cdots C_{n}\right)^{T} \\
\alpha_{\mathrm{I}}=\operatorname{diag}\left[\alpha_{n+1} \cdots \alpha_{i} \cdots \alpha_{m}\right] \\
\boldsymbol{F}_{\mathrm{IS}}=\left[F_{(n+1) \mathrm{S}} \cdots F_{i \mathrm{~S}} \cdots F_{m \mathrm{~S}}\right]^{T} \\
\boldsymbol{D}_{\mathrm{IS}}=\left[D_{(n+1) \mathrm{S}} \cdots D_{i \mathrm{~S}} \cdots D_{m \mathrm{~s}}\right]^{T} \\
\boldsymbol{F}_{\mathrm{II}}=\left[F_{(n+1) !} \cdots F_{i 1} \cdots F_{m \mathrm{I}}\right]^{T} \\
\boldsymbol{D}_{\mathrm{II}}=\left[D_{(n+1) 1} \cdots D_{i 1} \cdots D_{m \mathrm{I}}\right]^{T} \\
\boldsymbol{C}_{\mathrm{I}}=\left(C_{n+1} \cdots C_{i} \cdots C_{m}\right)^{T} .
\end{gathered}
$$


Only those elements on $\Gamma_{\mathrm{S}}$ have contribution to $\boldsymbol{F}_{i \mathrm{I}}, \boldsymbol{D}_{i \mathrm{I}}$.

Substituting (3.8)-(3.11) into (3.5), we obtain

$\frac{\alpha_{i}}{2 \pi} U_{\mathrm{i}}^{(1)}=\boldsymbol{F}_{i \mathrm{~S}} \boldsymbol{U}_{\mathrm{S}}^{(1)}+\boldsymbol{D}_{i \mathrm{~S}}\left(\frac{\partial \boldsymbol{U}^{(1)}}{\partial \boldsymbol{n}}\right)_{\mathrm{S}}+\boldsymbol{F}_{i \mathrm{I}} \boldsymbol{U}_{\mathrm{I}}^{(1)}+\boldsymbol{D}_{i \mathrm{I}}\left(\frac{\partial \boldsymbol{U}^{(1)}}{\partial \boldsymbol{n}}\right)_{\mathrm{I}}+C_{i}$.

For each node we can derive an equation as above. From all the nodes $i(1 \leqslant i \leqslant n)$ on $\Gamma_{\mathrm{s}}$, we derive an equation system:

$$
\frac{\alpha_{\mathrm{S}}}{2 \pi} \boldsymbol{U}_{\mathrm{S}}^{(1)}=\boldsymbol{F}_{\mathrm{SS}} \boldsymbol{U}_{\mathrm{S}}^{(1)}+\boldsymbol{D}_{\mathrm{SS}}\left(\frac{\partial \boldsymbol{U}^{(1)}}{\partial \boldsymbol{n}}\right)_{\mathrm{S}}+\boldsymbol{F}_{\mathrm{SI}} \boldsymbol{U}_{\mathrm{I}}^{(1)}+\boldsymbol{D}_{\mathrm{SI}}\left(\frac{\partial \boldsymbol{U}^{(1)}}{\partial \boldsymbol{n}}\right)_{\mathrm{I}}+C_{\mathrm{S}}
$$

From all the nodes $i(n+1 \leq i \leq m)$ on $\Gamma_{\mathrm{I}}$ we derive another equation system:

$$
\frac{\alpha_{\mathrm{I}}}{2 \pi} U_{\mathrm{I}}^{(1)}=\boldsymbol{F}_{\mathrm{IS}} \boldsymbol{U}_{\mathrm{S}}^{(1)}+\boldsymbol{D}_{\mathrm{IS}}\left(\frac{\partial \boldsymbol{U}^{(1)}}{\partial \boldsymbol{n}}\right)_{\mathrm{S}}+\boldsymbol{F}_{\mathrm{II}} \boldsymbol{U}_{\mathrm{I}}^{(1)}+\boldsymbol{D}_{\mathrm{II}}\left(\frac{\partial \boldsymbol{U}^{(1)}}{\partial \boldsymbol{n}}\right)_{\mathrm{I}}+C_{\mathrm{I}},
$$

where

$$
\begin{gathered}
\alpha_{\mathrm{S}}=\operatorname{diag}\left[\alpha_{1} \cdots \alpha_{i} \cdots \alpha_{n}\right] \\
\boldsymbol{F}_{\mathrm{SS}}=\left[F_{1 \mathrm{~S}} \cdots F_{i \mathrm{~s}} \cdots F_{n \mathrm{~s}}\right]^{T} \\
\boldsymbol{D}_{\mathrm{SS}}=\left[D_{1 s} \cdots D_{i \mathrm{~S}} \cdots D_{n \mathrm{~S}}\right]^{T} \\
\boldsymbol{F}_{\mathrm{SI}}=\left[F_{11} \cdots F_{i \mathrm{I}} \cdots F_{n \mathrm{I}}\right]^{T} \\
\boldsymbol{D}_{\mathrm{SI}}=\left[D_{11} \cdots D_{i 1} \cdots D_{n \mathrm{I}}\right]^{T} \\
\boldsymbol{C}_{\mathrm{S}}=\left(C_{1} \cdots C_{i} \cdots C_{n}\right)^{T} \\
\alpha_{\mathrm{I}}=\operatorname{diag}\left[\alpha_{n+1} \cdots \alpha_{i} \cdots \alpha_{m}\right] \\
\boldsymbol{F}_{\mathrm{IS}}=\left[F_{(n+1) \mathrm{S}} \cdots F_{i \mathrm{~S}} \cdots F_{m \mathrm{~S}}\right]^{T} \\
\boldsymbol{D}_{\mathrm{IS}}=\left[D_{(n+1) \mathrm{S}} \cdots D_{i \mathrm{~S}} \cdots D_{m \mathrm{~s}}\right]^{T} \\
\boldsymbol{F}_{\mathrm{II}}=\left[F_{(n+1) !} \cdots F_{i 1} \cdots F_{m \mathrm{I}}\right]^{T} \\
\boldsymbol{D}_{\mathrm{II}}=\left[D_{(n+1) 1} \cdots D_{i 1} \cdots D_{m \mathrm{I}}\right]^{T} \\
\boldsymbol{C}_{\mathrm{I}}=\left(C_{n+1} \cdots C_{i} \cdots C_{m}\right)^{T} .
\end{gathered}
$$


$E_{x}$ in region $\Omega_{1}, \Omega_{2}, \Omega_{3}$, respectively. For region $\Omega_{3}, U^{(3)}(P)$ at point $P$ on $\Gamma_{\mathrm{S}}$ is derived from (2.5) as

$$
\begin{aligned}
\frac{\alpha_{\mathrm{p}}^{\prime}}{2 \pi} U^{(3)}(P) & =\oint\left(U^{(3)} \frac{\partial \phi}{\partial n}-\phi \frac{\partial U^{(3)}}{\partial n}\right) \mathrm{d} \Gamma \\
& =\left(\int_{\Gamma_{\infty}^{\prime}}+\int_{\Gamma_{\mathrm{S}}}\right)\left(U^{(3)} \frac{\partial \phi}{\partial n}-\phi \frac{\partial U^{(3)}}{\partial n}\right) \mathrm{d} \Gamma,
\end{aligned}
$$

where $\alpha_{\mathrm{p}}^{\prime}$ is the angle subtended by $\Omega_{3}$ at $P$. Because the normal vector is defined inwards (Fig. 3), there is no negative sign before the integral of (4.1). The fundamental solution in $\Omega_{3}$ is $\phi=-(1 / 4) N_{0}\left(k_{3} r\right)$, where $k_{3}=\sqrt{\omega^{2} \mu \varepsilon}$. Differentiating $\phi$ partially with respect to $n$, we obtain

$$
\frac{\partial \phi}{\partial n}=\frac{\partial \phi}{\partial r} \frac{\partial r}{\partial n}=\frac{k_{3}}{4} N_{1}\left(k_{3} r\right) \cos \beta
$$

The anomalous field on $\Gamma_{\infty}^{\prime}$ is considered to be zero. From (1.17),

$$
\begin{aligned}
\left.U^{(3)}\right|_{\Gamma_{\infty}^{\prime}} & =\cos (\omega \sqrt{\mu \varepsilon} z)-(1-i) \sqrt{\frac{\sigma_{1}}{2 \omega \varepsilon}} \sin (\omega \sqrt{\mu \varepsilon} z) \\
& =\cos \left(k_{3} z\right)+i \frac{k_{1}}{k_{3}} \sin \left(k_{3} z\right)
\end{aligned}
$$

where

$$
\begin{gathered}
k_{1}=\sqrt{i \omega \mu \sigma_{1}} \\
\left.\frac{\partial U^{(3)}}{\partial n}\right|_{\Gamma_{\infty}^{\prime}}=\left.\left(\frac{\partial U^{(3)}}{\partial z} \frac{\partial z}{\partial n}\right)\right|_{\Gamma_{\infty}^{\prime}}=-\left[k_{3} \sin \left(k_{3} z\right)+k_{1} \cos \left(k_{3} z\right)\right] \cos (n, z),
\end{gathered}
$$

where $(n, z)$ is the angle between vector $n$ and the $z$-axis. So the integral on $\Gamma_{\infty}^{\prime}$ is given as

$$
\begin{gathered}
\int_{\Gamma^{\prime} \infty}\left(U^{(3)} \frac{\partial \phi}{\partial n}-\phi \frac{\partial U^{(3)}}{\partial n}\right) \mathrm{d} \Gamma=\int_{\Gamma_{\infty}^{\prime}} \frac{1}{4}\left[\left(k_{3} \cos \left(k_{3} z\right)-k_{1} \sin \left(k_{3} z\right)\right] N_{1}\left(k_{3} r\right) \cos \beta \mathrm{d} \Gamma\right. \\
-\int_{\Gamma_{\infty}^{\prime}} \frac{1}{4}\left[k_{3} \sin \left(k_{3} z\right)+k_{1} \cos \left(k_{3} z\right)\right] N_{0}\left(k_{3} r\right) \cos (n, z) \mathrm{d} \Gamma
\end{gathered}
$$

Dividing $\Gamma_{\infty}^{\prime}$ into elements, the above integral can be calculated with the Gaussian quadrature formula. It is a function of point $P$ and denoted as $A_{\mathrm{p}}$. Equation (4.1) can be written as 


$$
\frac{\alpha_{\mathrm{p}}^{\prime}}{2 \pi} U^{(3)}(P)=\frac{1}{4} \int_{\Gamma_{\mathrm{s}}} U^{(3)} k_{3} N_{1}\left(k_{3} r\right) \cos \beta \mathrm{d} \Gamma+\frac{1}{4} \int_{\Gamma_{\mathrm{s}}} \frac{\partial U^{(3)}}{\partial n} N_{0}\left(k_{3} r\right) \mathrm{d} \Gamma+A_{\mathrm{p}} .
$$

Considering the boundary condition (1.17) and using $U^{(1)}$ and $\partial U^{(1)} / \partial n$ to substitute $U^{(3)}$ and $\partial U^{(3)} / \partial n$, the formula can be written as

$$
\frac{\alpha_{\mathrm{p}}^{\prime}}{2 \pi} U^{(1)}(P)=\frac{1}{4} \int_{\Gamma_{\mathrm{s}}} U^{(1)} k_{3} N_{1}\left(k_{3} r\right) \cos \beta \mathrm{d} \Gamma+\frac{1}{4} \int_{\Gamma_{\mathrm{s}}} \frac{\partial U^{(1)}}{\partial n} N_{0}\left(k_{3} r\right) \mathrm{d} \Gamma+A_{\mathrm{p}}
$$

We decompose the above integral as a sum of integrals over each element. For node $i$, the above formula can be rewritten as

$$
\frac{\alpha_{i}^{\prime}}{2 \pi} U_{i}^{(1)}=\sum_{\Gamma_{\mathrm{s}}} \frac{1}{4} \int_{\Gamma_{\mathrm{E}}} U^{(1)} k_{3} N_{1}\left(k_{3} r\right) \cos \beta \mathrm{d} \Gamma+\sum_{\Gamma_{\mathrm{s}}} \frac{1}{4} \int_{\Gamma_{\mathrm{E}}} \frac{\partial U^{(1)}}{\partial n} N_{0}\left(k_{3} r\right) \mathrm{d} \Gamma+A_{i} .
$$

In a manner similar to (3.6) and (3.7), the integral on an element is derived as

$$
\begin{aligned}
& \int_{\Gamma_{\mathrm{E}}} U^{(1)} \frac{k_{3}}{4} N_{1}\left(k_{3} r\right) \cos \beta \mathrm{d} \Gamma \\
& =\int_{\Gamma_{\mathrm{E}}}\left[\begin{array}{ll}
\xi_{j} & \xi_{k}
\end{array}\right] \frac{k_{3}}{4} N_{1}\left(k_{3} r\right) \cos \beta \mathrm{d} \Gamma \cdot\left[\begin{array}{ll}
U_{j}^{(1)} & U_{k}^{(1)}
\end{array}\right]^{T}=\left[\begin{array}{ll}
g_{i j} & g_{i k}
\end{array}\right]\left[\begin{array}{ll}
U_{j}^{(1)} & U_{k}^{(1)}
\end{array}\right]^{T} \\
& \int_{\Gamma_{\mathrm{E}}} \frac{\partial U^{(1)}}{\partial n} \frac{1}{4} N_{0}\left(k_{3} r\right) \mathrm{d} \Gamma=\int_{\Gamma_{\mathrm{E}}}\left[\begin{array}{ll}
\xi_{j} & \xi_{k}
\end{array}\right] \frac{1}{4} N_{0}\left(k_{3} r\right) \mathrm{d} \Gamma \cdot\left[\left(\frac{\partial U^{(1)}}{\partial n}\right)_{j}\left(\frac{\partial U^{(1)}}{\partial n}\right)_{k}\right]^{T} \\
& =\left[\begin{array}{ll}
e_{i j} & e_{i k}
\end{array}\right]\left[\left(\frac{\partial U^{(1)}}{\partial n}\right)_{j}\left(\frac{\partial U^{(1)}}{\partial n}\right)_{k}\right]^{T} \text {, }
\end{aligned}
$$

where

$$
\begin{gathered}
g_{i j}=\int_{\Gamma_{\mathrm{E}}} \xi_{j} \frac{k_{3}}{4} N_{1}\left(k_{3} r\right) \cos \beta \mathrm{d} \Gamma, \\
g_{i k}=\int_{\Gamma_{\mathrm{E}}} \xi_{k} \frac{k_{3}}{4} N_{1}\left(k_{3} r\right) \cos \beta \mathrm{d} \Gamma, \\
e_{i j}=\frac{1}{4} \int_{\Gamma_{\mathrm{E}}} \xi_{j} N_{0}\left(k_{3} r\right) \mathrm{d} \Gamma, \\
e_{i k}=\frac{1}{4} \int_{\Gamma_{\mathrm{E}}} \xi_{k} N_{0}\left(k_{3} r\right) \mathrm{d} \Gamma .
\end{gathered}
$$


These four integrals can be calculated with the Gaussian quadrature formula. The sum of integrals over elements on $\Gamma_{\mathrm{S}}$ is

$$
\begin{aligned}
\sum_{\Gamma_{\mathrm{S}}} \frac{1}{4} \int_{\Gamma_{\mathrm{E}}} U^{(1)} k_{3} N_{1}\left(k_{3} r\right) \cos \beta \mathrm{d} \Gamma=\left[G_{i 1} \cdots G_{i j} \cdots G_{i n}\right]\left[U_{1}^{(1)} \cdots U_{j}^{(1)} \cdots U_{n}^{(1)}\right]^{T}=\boldsymbol{G}_{i \mathrm{~S}} \boldsymbol{U}_{\mathrm{S}}^{(1)} \\
\begin{aligned}
\sum_{\Gamma_{\mathrm{S}}} \frac{1}{4} \int_{\Gamma_{\mathrm{E}}} \frac{\partial U^{(1)}}{\partial n} N_{0}\left(k_{3} r\right) \mathrm{d} \Gamma & =\left[E_{i 1} \cdots E_{i j} \cdots E_{i n}\right]\left[\left(\frac{\partial U^{(1)}}{\partial n}\right)_{1} \cdots\left(\frac{\partial U^{(1)}}{\partial n}\right)_{j} \cdots\left(\frac{\partial U^{(1)}}{\partial n}\right)_{n}\right]^{T} \\
& =\boldsymbol{E}_{i \mathrm{~S}}\left(\frac{\partial \boldsymbol{U}^{(1)}}{\partial \boldsymbol{n}}\right)_{\mathrm{S}}
\end{aligned}
\end{aligned}
$$

where $G_{i j}$ and $E_{i j}$ are the sum of $g_{i j}, e_{i j}$ over elements around node $j$ :

$$
\begin{aligned}
\boldsymbol{G}_{i \mathrm{~S}} & =\left[G_{i 1} \cdots G_{i n}\right], \\
\boldsymbol{E}_{i \mathrm{~S}} & =\left[E_{i 1} \cdots E_{i n}\right] .
\end{aligned}
$$

Substituting (4.3) and (4.4) into (4.2), we obtain

$$
\frac{\alpha_{i}^{\prime}}{2 \pi} U_{i}^{(1)}=\boldsymbol{G}_{i \mathrm{~S}} \boldsymbol{U}_{\mathrm{S}}^{(1)}+\boldsymbol{E}_{i \mathrm{~S}}\left(\frac{\partial \boldsymbol{U}^{(1)}}{\partial \boldsymbol{n}}\right)_{\mathrm{S}}+A_{i} .
$$

For each node $i$ on $\Gamma_{\mathrm{s}}$, we can derive an equation as above. We now have the equation system for all nodes:

$$
\frac{\alpha_{\mathrm{S}}^{\prime}}{2 \pi} \boldsymbol{U}_{\mathrm{s}}^{(1)}=\boldsymbol{G}_{\mathrm{SS}} \boldsymbol{U}_{\mathrm{S}}^{(1)}+\boldsymbol{E}_{\mathrm{SS}}\left(\frac{\partial \boldsymbol{U}^{(1)}}{\partial \boldsymbol{n}}\right)_{\mathrm{S}}+A_{s}
$$

where

$$
\begin{aligned}
\alpha_{\mathrm{S}}^{\prime} & =\operatorname{diag}\left[\alpha^{\prime} \cdots \alpha^{\prime}{ }_{n}\right], \\
\boldsymbol{G}_{\mathrm{SS}} & =\left[G_{1 \mathrm{~S}} \cdots G_{n \mathrm{~S}}\right]^{T}, \\
\boldsymbol{E}_{\mathrm{SS}} & =\left[E_{1 \mathrm{~S}} \cdots E_{n \mathrm{~S}}\right]^{T}, \\
\boldsymbol{A}_{\mathrm{S}} & =\left(A_{1} \cdots A_{n}\right)^{T} .
\end{aligned}
$$

The equations in $\Omega_{1}$ and $\Omega_{2}$ are almost the same as in the $H_{x}$ polarization case. In $\Omega_{1}$, we have the same two equation systems as (3.13) and (3.14). Considering the boundary condition of $E_{x}$ polarization $\partial U^{(1)} / \partial n=\partial U^{(2)} / \partial n$, we obtain an equation system corresponding to (3.15):

$$
\boldsymbol{U}_{\mathrm{I}}^{(1)}=-\boldsymbol{F}_{\mathrm{II}}^{\prime} \boldsymbol{U}_{\mathrm{I}}^{(1)}-\boldsymbol{D}_{\mathrm{II}}^{\prime}\left(\frac{\partial \boldsymbol{U}^{(1)}}{\partial \boldsymbol{n}}\right)_{\mathrm{I}} .
$$


Combining (3.13), (3.14), (4.5) and (4.6), we derive a linear equation system:

$$
\left(\begin{array}{cccc}
\boldsymbol{F}_{\mathrm{SS}}-\frac{\alpha_{\mathrm{S}}}{2 \pi} & \boldsymbol{F}_{\mathrm{SI}} & \boldsymbol{D}_{\mathrm{SS}} & \boldsymbol{D}_{\mathrm{SI}} \\
\boldsymbol{F}_{\mathrm{IS}} & \boldsymbol{F}_{\mathrm{II}}-\frac{\alpha_{\mathrm{I}}}{2 \pi} & \boldsymbol{D}_{\mathrm{IS}} & \boldsymbol{D}_{\mathrm{II}} \\
\boldsymbol{G}_{\mathrm{SS}}-\frac{\alpha_{\mathrm{S}}}{2 \pi} & 0 & \boldsymbol{E}_{\mathrm{SS}} & 0 \\
0 & \boldsymbol{F}_{\mathrm{II}}^{\prime}+I-\frac{\alpha_{\mathrm{I}}^{\prime}}{2 \pi} & 0 & \boldsymbol{D}_{\mathrm{II}}^{\prime}
\end{array}\right)\left(\begin{array}{c}
\boldsymbol{U}_{\mathrm{S}}^{(1)} \\
\boldsymbol{U}_{\mathrm{I}}^{(1)} \\
\left(\frac{\partial \boldsymbol{U}^{(1)}}{\partial \boldsymbol{n}}\right)_{\mathrm{S}} \\
\left(\frac{\partial \boldsymbol{U}^{(1)}}{\partial \boldsymbol{n}}\right)_{\mathrm{I}}
\end{array}\right)=\left(\begin{array}{c}
-\boldsymbol{C}_{\mathrm{S}} \\
-\boldsymbol{C}_{\mathrm{I}} \\
-\boldsymbol{A}_{\mathrm{S}} \\
0
\end{array}\right) .
$$

We have $2 m$ equations in total. The number of unknowns is also $2 m$. We resolve this equation to derive the values of $U$ (that is: $E_{x}$ ) and $\partial U / \partial n$ on the ground surface. Substituting $E_{x}=U, H_{y}=(1 / \mathrm{i} \omega \mu) \partial E_{x} / \partial z=-(1 / \mathrm{i} \omega \mu) \partial U / \partial n$ into (1.2), the apparent resistivity can be found.

\section{Examples}

\section{$6.1 H_{x}$ polarization}

Figure 5 shows a syncline model. The resistivity of surrounding rock is $\rho_{1}=20$ $\Omega \mathrm{m}$, and of syncline is $\rho_{2}=1 \Omega \mathrm{m}$. We calculated the magnetotelluric profile for a
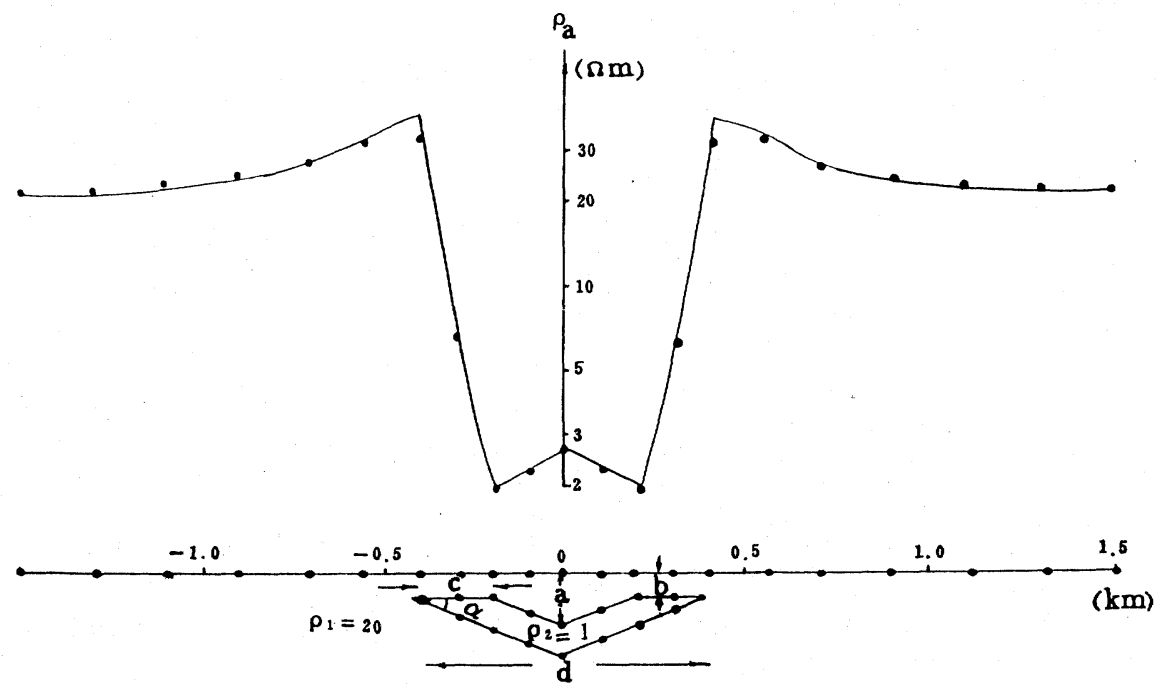

Fig. 5. The magnetotelluric profile for $H_{x}$ polarization for $T=10 \mathrm{sec}$. The curve indicates the result of the boundary element method, while dots indicate the result of the finit element method. Model parameters are $a=140 \mathrm{~m}, b=70 \mathrm{~m}, c=200 \mathrm{~m}, d=800 \mathrm{~m}, \alpha=19.3^{\circ}$. 
period $T=10 \mathrm{~s}$. The wavelength of the electromagnetic wave in surrounding rock is $\lambda_{1}=\sqrt{10 \rho_{1} T}=44.7 \mathrm{~km}$, and in syncline is $\lambda_{2}=10 \mathrm{~km}$. The locations of nodes are shown in Fig. 5. The number of nodes on the ground surface is 29 ( 8 nodes located beyond $\pm 1.5 \mathrm{~km}$ are not shown in Fig. 5) and on the conductivity boundary is 16 . The length of element is less than $\lambda_{1} / 8$ and $\lambda_{2} / 8$. The curve in the upper half of Fig. 5 is the magnetotelluric profile calculated by the boundary element method and the points near the curve are the result by the finite element method. The results by both methods are in good agreement.

\section{$6.2 E_{x}$ polarization}

Figure 6 is an erect thick plate model. The resistivity of surrounding rock is $\rho_{1}=10 \Omega \mathrm{m}$, and of plate is $\rho_{2}=1 \Omega \mathrm{m}$. We calculated the magnetotelluric profile for a period $T=1 \mathrm{~s}$. The wavelength of the electromagnetic wave in surrounding rock is $\lambda_{1}=\sqrt{10 \rho_{1} T}=10 \mathrm{~km}$, and in plate is $\lambda_{2}=3.16 \mathrm{~km}$. The locations of nodes are shown in Fig. 6 . The number of nodes on the ground surface is 17 ( 6 nodes located beyond \pm 2 $\mathrm{km}$ are not shown in Fig. 6) and on the conductivity boundary is 12. The length of element is less than $\lambda_{1} / 8$ and $\lambda_{2} / 8$. The curve in the upper half of Fig. 6 is the magnetotelluric profile calculated by the boundary element method and the points near the curve are the result by the finite element method.

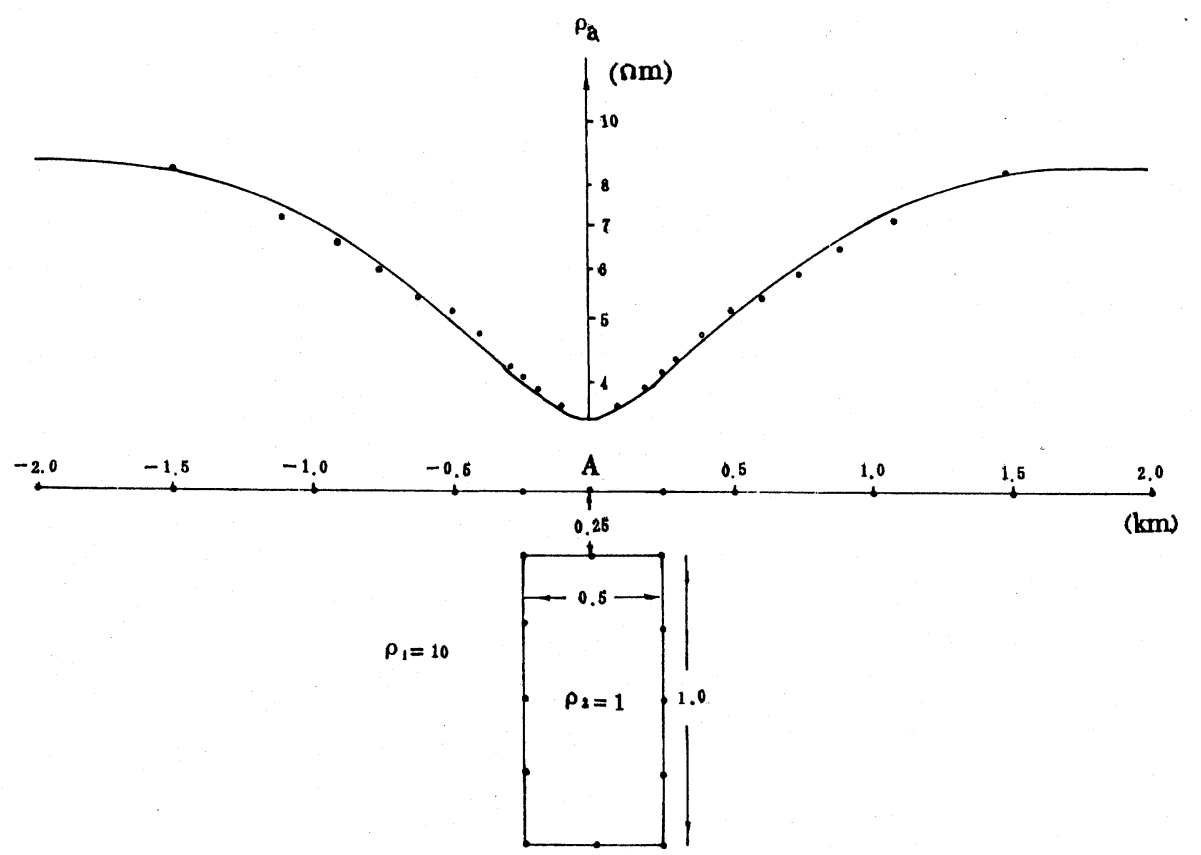

Fig. 6. The magnetotelluric profile of $E_{x}$ polarization for $T=1 \sec$ (一:the result of the boundary element method. $\because$ the result of the finite element method). 
Figure 7 is the magnetotelluric response curve at point A in Fig. 6. The curve is the result of the boundary element method and the points represent the result of the finite element method. From Figs. 5, 6 and 7 it is obvious that the results of the boundary element method are identical to those of the finite element method. This shows that the boundary element method is effective.

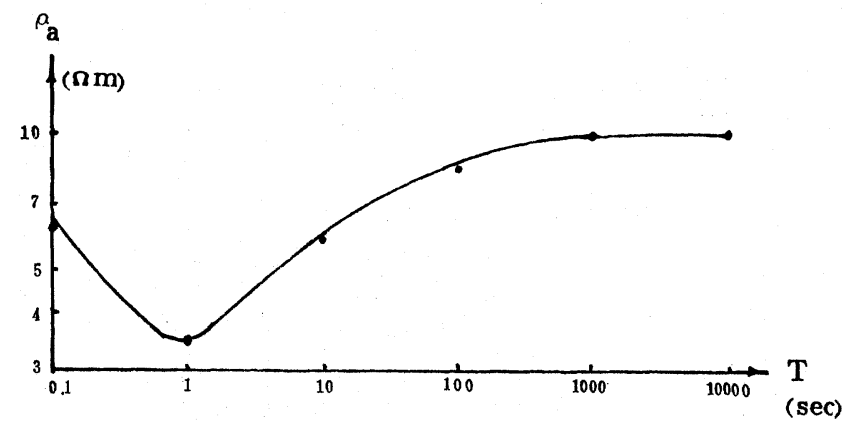

Fig. 7. The magnetotelluric sounding curve at point A (see Fig. 6) (-:the result of boundary element method. $\because$ the result of the finite element method).

\section{Conclusion}

In the finite element method, element division is made over the whole domain. When the anomalous body is of complicated shape, this element division and initial data preparation are tedious. In the boundary element method, only the boundary needs to be divided, so the division is easier. It is also possible to use more elements to approximate the boundary more precisely. In addition, the boundary element method needs far less memory space, so it can run even on a microcomputer. The examples given above show that the results obtained by both methods are very similar. In particular, the boundary element method is suitable for a model with one anomalous body. When there are several anomalous bodies, derivation of the formula and programing are complicated.

The authors are grateful to Dr. D. W. Strangway for his help in preparing this manuscript and to Dr. Y. Honkura for his revising the original manuscript.

Appendix: The Integration of $\delta$ Function on an Area

Let $p$ be a fixed point on a plain, $q$ a moving point and $\varepsilon$ a small circle enclosing $p$. We define 


$$
\delta_{\varepsilon}(q, p)= \begin{cases}\frac{1}{\varepsilon}, & q \in \varepsilon \\ 0, & q \notin \varepsilon\end{cases}
$$

and

$$
\delta(p)=\lim _{\varepsilon \rightarrow 0} \delta_{\varepsilon}(q, p)=\left\{\begin{array}{c}
\infty, q=p \\
0, q \neq p .
\end{array}\right.
$$

Then $\delta(p)$ is a 2-D $\delta$ function centered at $p$. Let $\Omega$ represent a 2-D domain and $\Gamma$ be the boundary of $\Omega$. When $p$ is on $\Gamma$ (Fig. 2), $\varepsilon$ is divided into two parts. Let $\varepsilon^{\prime}$ represent the part within $\Omega$. Then

$$
\int_{\Omega} \delta(p) \mathrm{d} \Omega=\lim _{\varepsilon \rightarrow 0} \int_{\varepsilon^{\prime}} \delta(p) \mathrm{d} \Omega=\lim _{\varepsilon \rightarrow 0} \int_{\varepsilon^{\prime}} \frac{\mathrm{d} \Omega}{\varepsilon}=\lim _{\varepsilon \rightarrow 0} \frac{\varepsilon^{\prime}}{\varepsilon}=\frac{\alpha_{\mathrm{p}}}{2 \pi}
$$

where $\alpha_{p}$ is the angle subtended by $\Omega$ at $p$. If $U$ is a continuous function of $p$, then

$$
\int_{\Omega} U \delta(p) \mathrm{d} \Omega=\lim _{\varepsilon \rightarrow 0} \int_{\varepsilon^{\prime}} U \delta(p) \mathrm{d} \Omega=U(p) \lim _{\varepsilon \rightarrow 0} \int_{\varepsilon^{\prime}} \delta(p) \mathrm{d} \Omega=\frac{\alpha_{p}}{2 \pi} U(p) .
$$

\section{REFERENCES}

Brebbia, C. A., The Boundary Element Method for Engineers, 188 pp., Pentech Press, London, 1978.

Brynelli, B. E., M. A. Dobrovol'skaya, A. A. Kovtun, and N. S. Kuznetsov, Magnito-telluricheskoe profilirovanie na modelyakh gorizontalno neodnorodnynch struktur (Magnetotelluric profiling over models with horizontal-inhomogeneous structure)-Novya metody elektricheskoi razvedki na neft $\mathrm{i}$ gas, Gostoptechizdat, 114-119, 1963.

Brynelli, B. E., M. A. Dobrovol'skaya, A. A. Kovtun, and N. S. Kuznetsov, Nekotorye rezultaty MTP i MTZ na modelyach neodnorodnych struktur (Some results of MTP and MTS over models of inhomogeneous structure)-Magnito-telluricheskie metody izucheniya stroeniya zemnoi kory i verchnei mantii: Acad. of Sci. of the UDSSR, Soviet Geophys. Committee, 4, 125-129, 1969.

CAGniard, L., Basic theory of the magnetotelluric method of geophysical prospecting, Geophysics, 18 , 605-635, 1953.

Coggon, J. H., Electromagnetic and electrical modeling by the finite element method, Geophysics, 36, $132-155,1971$.

Dosso, H. W., Analogue model measurements for electromagnetic variations near vertical faults and dikes, Canad. J. Earth Sci., 3, 287-325, 1966.

DMITRIYeV, V. I. and Ye. V. ZAKHAROV, A method for solving problems on the electrodynamics of inhomogeneous media, J. Comput. Math. Math. Phys., 24, 1458-1464, 1970.

D'ERCEVILLE, I. and G. KUNETS, The effect of a fault on the earth's electromagnetic field, Geophysics, 27, $651-655,1962$.

PARRY, J.R. and S. H. WARD, Electromagnetic scattering from cylinders of arbitrary cross-section in a conductive half-space, Geophysics, 36, 67-100, 1971.

RANKIN, D., The magnetotelluric effect on a dike, Geophysics, 27, 666-676, 1962.

REDDY, I. K. and D. RANKIN, Magnetotelluric response of a two-dimensional sloping contact by the finite element method, Tageoph., 105, 847-857, 1973. 
TAHERI, F., Interpretation de mesures du champ tellurique an moyen de reseaux analogiques a resistances et capacities (Interpretation of measurements of the telluric field by R-C-analog-models), These a la faculte des sciences de Paris, 1968.

$\mathrm{XU}, \mathrm{S}$. Z. and S. K. ZHAO, Solution of magnetotelluric field equations for a two-dimensional anisotropic geoelectric section by the finite element method, Acta Seismologica Sinica, 7, 80-90, 1985.

ZAKHAROV, Ye. V., Method of solution of problems in magnetotelluric sounding (MTS) theory for a medium with horizontally periodic structure, Earth Physics, No. 10, 53-56, 1975.

ZHAO, S. K. and S. Z. Xu, Two-dimensional magnetotelluric modeling by finite element method, Comput. Tech. Geophys. Geochem. Explor., No. 1, 17-21, 1983. 